\title{
Scattering database for spheroidal particles
}

\author{
Karsten Schmidt, ${ }^{1, \star}$ Jochen Wauer, ${ }^{2}$ Tom Rother, ${ }^{1}$ and Thomas Trautmann ${ }^{3}$ \\ ${ }^{1}$ Remote Sensing Technology Institute, German Aerospace Center, Kalkhorstweg 53, D-17235 Neustrelitz, Germany \\ ${ }^{2}$ Institute for Meteorology, University of Leipzig, Stephanstrasse 3, D-04103 Leipzig, Germany \\ ${ }^{3}$ Remote Sensing Technology Institute, German Aerospace Center, Münchner Straße 20, D-82234 Wessling, Germany \\ ${ }^{*}$ Corresponding author: Karsten.Schmidt@dlr.de
}

Received 29 January 2009; accepted 23 February 2009;

posted 16 March 2009 (Doc. ID 106910); published 6 April 2009

\begin{abstract}
We present a database containing light scattering quantities of randomly oriented dielectric spheroidal particles in the resonance region. The database has been generated by using a thoroughly tested $T$-matrix method implementation. The data possess a defined accuracy so that they can be used as benchmarks for electromagnetic and light scattering computations of spheroids. Within its parameter range the database may also be applied as a fast tool to investigate the scattering properties of nonspherical particles and to verify assumptions or statements concerning their scattering behavior. A user interface has been developed to facilitate the data access. It also provides some additional functionalities such as interpolations between data or the computation of size-averaged scattering quantities. A detailed description of the database and the user interface is given, followed by examples illustrating their capabilities and handling. On request, the database including the documentation is available, free of charge, on a CD-ROM. (C) 2009 Optical Society of America

OCIS codes: $\quad 290.0290,290.5850$.
\end{abstract}

\section{Introduction}

Scattering of electromagnetic waves on nonspherical dielectric particles becomes of growing importance in remote sensing of the Earth's atmosphere as well as in technical and medical diagnostics. Studying the influence of dustlike particles on our climate system is only one example in this context, which is of particular interest to us. For instance, Markowicz et al. [1] pointed out that ground-based interferometer observations in the Cape Verde region clearly show the signal of Saharan mineral dust particles in the atmospheric infrared window region. Since the typical size of airborne dust particles is in the range between $2 \mu \mathrm{m}$ and $10 \mu \mathrm{m}$, it can be expected that particle scattering is most sensitive to particle shape in the thermal infrared window region. The impact of the shape of mineral dust particles on the transmitted and reflected solar radiation has been recently investigated during the Saharan Mineral Dust Experiment

0003-6935/09/112154-11\$15.00/0

(C) 2009 Optical Society of America
(SAMUM). Otto et al. [2] showed that modeled downwelling spectral irradiances are less sensitive to the nonsphericity of assumed spheroidal mineral dust particles. But in contrast to this, prolate or oblate dust particles lead to a much stronger hemispheric backscattering of solar radiation as compared to a particle population consisting of surface-equivalent spheres with the same refractive indices.

Recent and upcoming instruments measure in wavelength regions on the order of the size of aerosols so that deviations from the spherical particle shape may represent major uncertainties in the data interpretation. For example, the balloon-borne Terahertz Limb Sounder (TELIS) instrument, operating in the range $500 \mathrm{GHz}$ to $1.8 \mathrm{THz}$, has the capability to detect particles from polar stratospheric clouds as well as from cirrus clouds. Scattering and emission from these particles interferes with the feature-rich emission spectra caused by various atmospheric trace gases. Therefore, for cirrus particles having effective radii ranging typically from $20 \mu \mathrm{m}$ to several $100 \mu \mathrm{m}$, shape and composition information will be key parameters that determine the single scattering 
properties of these particles in the far-infrared wavelength region.

The instruments Superconducting Submillimeterwave Limb-emission Sounder (SMILES; see Kasai et al. [3]) and First Light from the Far-Infrared Spectroscopy of the Troposphere (FIRST; see Mlynczak et al. [4]) are able to study many aspects of the Earth's climate, including water vapor feedbacks, cirrus radiative properties, and the natural greenhouse effect of the planet. SMILES is a new technology of submillimeter-wave sensors for sounding the middle atmosphere. Its operation is planned for 2009 aboard the International Space Station. SMILES will provide the scientific community with global data not only for trace gases but also for high-altitude clouds. Because the typical size of cirrus cloud particles is also in the submillimeter region, scattering on particles with nonspherical shapes will be a major ingredient for a physically consistent interpretation of spectra measured by instruments such as TELIS, SMILES, and FIRST (see also Emde et al. [5]).

Since scattering of electromagnetic waves on small particles represents a fundamental physical process, it plays an important role not only in remote sensing but also in many other fields of science and technology. Increasingly, modern optical measurement techniques have emerged that make it necessary to take nonspherical particles into account. However, there are two particular aspects that make this necessity a complex task. First, the numerical effort is much higher than that for spherical particles within Mie theory. This effort strongly depends on the morphology of the particle and can be performed online only in very specific situations. Second, the convergence procedures of the existing approaches are much more complex as compared to spheres. To obtain reliable results requires a detailed knowledge of the method behind a certain approach. Otherwise one can run into a lot of pitfalls.

Databases containing scattering quantities of nonspherical particles, either precalculated or experimentally determined, release the user to deal with these problems. They offer fast access to the data, which is important for, e.g., near real-time processing. Of course, effort and care have to be spent in advance when generating trustworthy data. Depending on the aim of the database, different data requirements result. For example, the database by Yang et al. [6] comprises complex ice particles to model the radiative properties of cirrus clouds in the nearthrough far-infrared spectral region. Dubovik et al. [7] considered spheroids to account for the aerosol particle nonsphericity in remote sensing of desert dust. In order to cope with the wide size parameter regions, different exact and approximate methods were used to fill both databases. The database by Volten et al. [8] contains experimental scattering matrix elements measured at $441.6 \mathrm{~nm}$ and $632.8 \mathrm{~nm}$ for a large collection of micrometer-sized mineral particles in random orientation to provide the light scattering community with easily accessible data that are useful for a variety of applications. Information on the accuracy of the data are given whenever available.

In the paper at hand we present a database containing light scattering quantities of randomly oriented dielctric spheroidal particles in the resonance region. Its primary aim is to provide benchmarks for electromagnetic and light scattering computations of spheroids. Thus the data were generated by using a thoroughly tested $T$-matrix method implementation [9] briefly outlined in Section 2. In doing so great store was set by a defined accuracy. Due to restrictions of our implementation [9] and due to the data accuracy aspired, we were bounded in the parameter range (see Section 3). But within the region covered the database may also be applied as a fast tool to investigate the scattering properties of nonspherical particles and to verify assumptions or statements concerning their scattering behavior. Of course, the database can be used in certain applications, too, if its parameters meet their requirements. The resolution with respect to the size parameter and the scattering angles of the phase function, discussed in detail in Section $\underline{3}$, allows for a reliable interpolation of all data within a defined accuracy. A user interface, described in Section 4, was developed to facilitate the data access. It also offers some additional functionalities such as the interpolations between data and the computation of size-averaged scattering quantities. In Section 5, examples are provided to illustrate the capabilities of the database and the handling of the user interface in the different operation modes.

\section{Method and Accuracy Aspects}

Some years ago Wauer et al. [9] presented two software tools to deal with the plane wave scattering on nonspherical particles. One of these tools, the program Mieschka, has been developed to treat axisymmetric scatterers in spherical coordinates. It is based on the T-matrix method of Waterman [10], which has been substantially advanced through the analytical orientation averaging and intensively investigated and applied by Mishchenko et al. (e.g., [11,12]). This program has been used to compute the data in the database. Within the $T$-matrix method all fields are expanded in terms of finite series of vector spherical wave functions $R g \Psi_{\tau n l}$ and $\Psi_{\tau n l}$, respectively. In particular the incident $\left(\mathbf{E}^{\text {inc }}\right)$ and the scattered $\left(\mathbf{E}^{\text {scat }}\right)$ field read as follows:

$$
\begin{gathered}
\mathbf{E}^{\mathrm{inc}}(k \mathbf{r})=\sum_{\tau=1}^{2} \sum_{l=-l_{\text {cut }}}^{l_{\text {cut }}} \sum_{n=|l|}^{n_{\text {cut }}} a_{\tau n l} \cdot R g \Psi_{\tau n l}(k \mathbf{r}), \\
\mathbf{E}^{\mathrm{scat}}(k \mathbf{r})=\sum_{\tau=1}^{2} \sum_{l=-l_{\text {cut }}}^{l_{\text {cut }}} \sum_{n=|l|}^{n_{\text {cut }}} f_{\tau n l} \cdot \Psi_{\tau n l}(k \mathbf{r}) .
\end{gathered}
$$

The expansion coefficients $f_{\tau n l}$ of the scattered field are calculated from the known incident field 
expansion coefficients $a_{\tau n l}$ by use of the $T$-matrix relation

$$
\mathbf{f}=\mathcal{T} \cdot \mathbf{a} .
$$

The $T$-matrix itself is the product of two matrices,

$$
\mathcal{T}=-\mathcal{Q}^{-1} \cdot \operatorname{Rg} \mathcal{Q},
$$

the elements of which are integrals over the scatterer boundary surface considered and contain combinations of the vector spherical wave functions (for more details see, e.g., $[9,10])$. In order to compute polarimetric scattering quantities, $\mathbf{E}^{\text {inc }}$ and $\mathbf{E}^{s}$ are decomposed into vertically $(v)$ and horizontally $(h)$ polarized parts with respect to the $x-z$ plane as the reference plane within a Cartesian coordinate system $(x, y, z)$. Considering an incident plane wave,

$$
\mathbf{E}^{\text {inc }}(k \mathbf{r})=\mathbf{e}_{0} \cdot \exp (i k z),
$$

propagating along the $z$-direction, the unit vector $\mathbf{e}_{0}$ characterizes its polarization state. In the case of a vertically polarized field, $\mathbf{e}_{0}$ is defined to be $\mathbf{e}_{\gamma}$. On the other hand, we set $\mathbf{e}_{0}=\mathbf{e}_{x}$ for a horizontally polarized incident field. $k=2 \pi / \lambda$ denotes the wavenumber of the incident plane wave with the wavelength $\lambda$. The scattered field polarization is appointed to be

$$
\begin{aligned}
& E_{v}^{s}=E_{\phi}^{s}, \\
& E_{h}^{s}=E_{\theta}^{s},
\end{aligned}
$$

in spherical coordinates $(r, \theta, \phi)$. The relation between the polarized scattered field in the far field approximation and the polarized incident field is governed by the scattering amplitude matrix $\mathcal{F}$ (see, e.g., $[\underline{13}, \underline{14}])$ :

$$
\left(\begin{array}{c}
E_{v}^{s} \\
E_{h}^{s}
\end{array}\right)=\frac{\exp (i k r)}{r} \cdot\left(\begin{array}{c}
F_{v v}(\theta, \phi) F_{v h}(\theta, \phi) \\
F_{h v}(\theta, \phi) F_{h h}(\theta, \phi)
\end{array}\right) \cdot\left(\begin{array}{l}
E_{v}^{\text {inc }} \\
E_{h}^{\text {inc }}
\end{array}\right) .
$$

Note that the azimuthal angle $\phi$ is either $0^{\circ}$ or $180^{\circ}$ in the reference plane. All scattering quantities of interest are computed on the basis of the scattering amplitudes $F_{\alpha \beta},(\alpha, \beta)=(v, h)$. If the particle is randomly oriented then orientation-averaged quantities are considered to characterize its scattering properties. Let $M$ be any scattering quantity. Its average $\langle M\rangle_{\text {or }}$ is defined according to

$$
\begin{aligned}
\langle M\rangle_{\text {or }}:= & \frac{1}{8 \pi^{2}} \cdot \int_{0}^{2 \pi} \mathrm{d} \phi_{p} \int_{0}^{\pi} \mathrm{d} \theta_{p} \sin \theta_{p} \\
& \int_{0}^{2 \pi} \mathrm{d} \psi_{p} M\left(\phi_{p}, \theta_{p}, \psi_{p}\right)
\end{aligned}
$$

with the Eulerian angles $\left(\phi_{p}, \theta_{p}, \psi_{p}\right)$ describing the orientation of the particle with respect to the incident plane wave. The orientation averaging can be accomplished either numerically or analytically according to, e.g., $[11,12,15]$. Note furthermore that efficiencies are defined to be the corresponding cross sections divided by the average geometrical cross section $q$ of the spheroid in random orientation. According to van de Hulst [13] the latter one is onefourth of the surface area.

The following scattering quantities were considered for the database:

- the orientation-averaged extinction efficiency via the optical theorem

$$
\sigma_{\text {eff }}^{\text {ext }}=\frac{\sigma^{\text {ext }}}{q}=\frac{4 \pi}{k q} \cdot\left\langle\operatorname{Im} F_{\alpha \alpha}\left(\theta=0^{\circ}, \phi=0^{\circ}\right)\right\rangle_{\text {or }}, \quad \alpha=(v, h),
$$

- the orientation-averaged (total) scattering efficiency

$$
\begin{aligned}
\sigma_{\text {eff }}^{\text {sca }}= & \frac{\sigma^{\text {sca }}}{q}=\frac{1}{q} \int_{0}^{2 \pi} \mathrm{d} \phi \int_{0}^{\pi} \mathrm{d} \theta \sin \theta\left(\left\langle\left|F_{\alpha \alpha}(\theta, \phi)\right|^{2}\right\rangle_{\text {or }}\right. \\
& \left.+\left\langle\left|F_{\beta \alpha}(\theta, \phi)\right|^{2}\right\rangle_{\text {or }}\right), \quad \alpha \neq \beta,
\end{aligned}
$$

- the orientation-averaged absorption efficiency

$$
\sigma_{\mathrm{eff}}^{\mathrm{abs}}=\frac{\sigma^{\mathrm{abs}}}{q}=\sigma_{\mathrm{eff}}^{\mathrm{ext}}-\sigma_{\mathrm{eff}}^{\mathrm{sca}},
$$

- the orientation-averaged single scattering albedo

$$
\omega=\frac{\sigma^{\mathrm{sca}}}{\sigma^{\mathrm{ext}}}
$$

[13]

the phase function according to van de Hulst

$$
\begin{aligned}
P(\theta)= & \frac{k^{2}}{2} \cdot\left(\left\langle\left|F_{h h}\left(\theta, \phi=0^{\circ} / 180^{\circ}\right)\right|^{2}\right\rangle_{\text {or }}\right. \\
& +\left\langle\left|F_{h v}\left(\theta, \phi=0^{\circ} / 180^{\circ}\right)\right|^{2}\right\rangle_{\text {or }} \\
& +\left\langle\left|F_{v h}\left(\theta, \phi=0^{\circ} / 180^{\circ}\right)\right|^{2}\right\rangle_{\text {or }} \\
& \left.+\left\langle\left|F_{v v}\left(\theta, \phi=0^{\circ} / 180^{\circ}\right)\right|^{2}\right\rangle_{\text {or }}\right),
\end{aligned}
$$

afterwards being normalized according to

const $\cdot \int_{0}^{\pi} \mathrm{d} \theta \sin \theta P(\theta)=\int_{0}^{\pi} \mathrm{d} \theta \sin \theta p(\theta)=2$,

- the asymmetry parameter

$$
g=\frac{1}{2} \int_{-1}^{1} \mathrm{~d} \cos \theta \cos \theta p(\theta)
$$


- the orientation-averaged direct-polarized backscattering efficiency

$$
\begin{aligned}
\sigma_{\text {dir,eff }}^{\text {back }} & =\frac{1}{q} \cdot \sigma_{\text {dir }}^{\text {back }}=\frac{k^{2}}{q} \cdot\left\langle\left|F_{\alpha \alpha}\left(\theta=180^{\circ}, \phi=0^{\circ}\right)\right|^{2}\right\rangle_{\mathrm{or}}, \\
\alpha & =(v, h),
\end{aligned}
$$

- the orientation-averaged cross-polarized backscattering efficiency

$$
\begin{aligned}
\sigma_{x, \text { eff }}^{\text {back }} & =\frac{1}{q} \cdot \sigma_{x}^{\text {back }}=\frac{k^{2}}{q} \cdot\left\langle\left|F_{\alpha \beta}\left(\theta=180^{\circ}, \phi=0^{\circ}\right)\right|^{2}\right\rangle_{\text {or }}, \\
\alpha & \neq \beta .
\end{aligned}
$$

From the numerical point of view there are four aspects that influence the accuracy of the computational results. These are

- the determination of the truncation parameters $n_{\text {cut }}$ and $l_{\text {cut }}$ of the expansions [Eqs. (1) and (2)],

- the performance of the surface integrations in the elements of the matrices $\mathcal{Q}$ and $\operatorname{Rg} \mathcal{Q}$ in Eq. (4),

- the performance of the inversion of the matrix $\mathcal{Q}$ in Eq. (4), and

- the performance of the orientation averaging procedure.

Detailed information about the procedures to deal with the above points and some benchmarks are provided in [9]. It should however be mentioned that the truncation value $n_{\text {cut }}$ was determined for a given spheroid by inspecting its differential scattering cross sections at a plane wave incidence along the rotational axis. If a maximum relative error of $2 \%$ was achieved at $80 \%$ of all scattering angles while $n_{\text {cut }}$ was increased then $n_{\text {cut }}$ was fixed to the current value. This convergence strategy follows an idea of Barber and Hill [16], and it has been approved in many of our applications, too. Once $n_{\text {cut }}$ was fixed $l_{\text {cut }}$ was determined by applying the above convergence strategy to an effective phase function averaged only over 9 different angles of orientation. The surface integrals forming the elements of the matrices $\mathcal{Q}$ and $\operatorname{Rg} \mathcal{Q}$ in Eq. (4) were computed with a maximum relative error of $0.1 \%$. Note finally that the program Mieschka has been tested in many applications and compared with results obtained by other software packages.

\section{Database Content and Parameter Range}

The database contains the orientation-averaged scattering quantities, listed in Section 2 , of spheroids. Their geometry is described in Cartesian coordinates by

$$
\left(\frac{x}{b}\right)^{2}+\left(\frac{y}{b}\right)^{2}+\left(\frac{z}{a}\right)^{2}=1 .
$$

Here the $z$ axis represents the rotational axis, and $a$ and $b$ denote the semi-axes. The data are stored in terms of the aspect ratio $a v=a / b$ and the size parameter $k r_{\text {eqv }(v)}$ of the volume-equivalent sphere with the radius $r_{\text {eqv }(v)}=\left(a b^{2}\right)^{1 / 3}$. An aspect ratio $a v>1$ belongs to prolate spheroids, and $a v<1$ corresponds to oblate ones. Table 1 summarizes the refractive indices $n$ and the aspect ratios $a v$ for which scattering data are present in the database. These values were chosen to fit into the needs of lidar measurements within SAMUM. The present resolutions with respect to $n$ and $a v$ do not allow for a reliable interpolation of the scattering data. To meet the predefined accuracy requirements the size parameter $k r_{\text {eqv }(v)}$ had to be restricted to the maximum value 40 .

\section{A. Resolution with Respect to the Size Parameter}

Regarding the resolution with respect to the size parameter, $\Delta k r_{\text {eqv }(v)}=0.2$ was chosen. This resolution enables interpolations between adjacent data sets and was determined by means of the backscattering efficiency. The latter represents a differential scattering quantity that is known to be very sensitive to any change of the particle morphology (size, shape, and refractive index), in general. Moreover it is of special importance in lidar measurements. Like other scattering quantities it exhibits, for nonspherical particles in random orientation, a much smoother behavior as a function of the size parameter than that of spheres. Thus the investigations were done on spheres as the worst case. The resolution obtained in this case was also used for the spheroids.

Figure 1 shows an example of the backscattering and the extinction efficiency of a sphere with a refractive index $n=1.33$ as a function of the size parameter at a resolution of $\Delta k r_{\text {eqv }(v)}=0.025$. It is seen that the backscattering efficiency has much stronger oscillations than the extinction efficiency, thus revealing its sensitivity mentioned above. Therefore we considered the backscattering efficiency for determining the size parameter resolution. The morphology-dependent resonances are seen in both plots. Since we do not intend to resolve the morphology-dependent resonances by interpolation we focused on the size parameter range up to 12.5 to determine $\Delta k r_{\text {eqv }(v)}$. Figure $\underline{2}$ provides an example of these investigations. It shows the backscattering efficiency of the sphere of Fig. 1 in the size parameter range [7.5,12.5] at different resolutions $\left(\Delta k r_{\text {eqv }(v)}=\right.$ $0.6,0.4$ and 0.2 ). It turned out that a resolution of $\Delta k r_{\text {eqv }(v)}=0.2$ is sufficient to reproduce the original reference curve, generated with $\Delta k r_{\text {eqv }(v)}=0.025$, by interpolation. The maximum error of $23 \%$ seen in Fig. 2(d) was only reached in the deepest troughs. In Section 5, Fig. 5, below, we will present the normalized phase function $p(\theta)$ of a randomly oriented spheroid with $n=1.8$ and $a v=1.5$ at a size

Table 1. Refractive Indices $n$ and Aspect Ratios av Present in the Database

\begin{tabular}{llllllll}
\hline $\operatorname{Re}(n)$ & 1.33 & 1.4 & 1.5 & 1.6 & 1.7 & 1.8 & \\
$\operatorname{Im}(n)$ & 0 & 0.001 & 0.005 & 0.01 & 0.03 & 0.05 & 0.1 \\
$a v$ & 0.67 & 0.77 & 0.87 & 1.0 & 1.15 & 1.3 & 1.5 \\
\hline
\end{tabular}




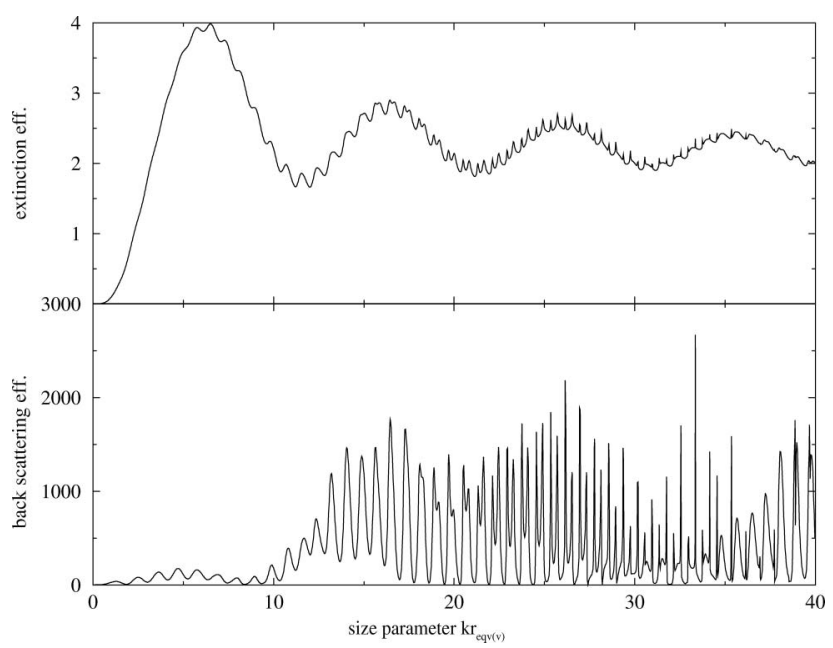

Fig. 1. Extinction efficiency $\sigma_{\text {eff }}^{\text {ext }}$ (top) and direct-polarized backscattering efficiency $\sigma_{\text {direff }}^{\text {back }}$ (bottom) as a function of the volumeequivalent size parameter $k r_{\text {eqv }(v)}$ for a spherical scatterer with a refractive index $n=1.33$. The resolution is $\Delta k r_{\operatorname{eqv}(v)}=0.025$.

parameter $k r_{\text {eqv }(v)}=39.52$. It is compared with a phase function obtained by a linear interpolation of the phase functions at $k r_{\text {eqv }(v)}=39.4$ and $k r_{\text {eqv }(v)}=39.6$. The agreement between both curves implies that the size parameter resolution $\Delta k r_{\text {eqv }(v)}=0.2$, originally determined within the size parameter range up to 12.5 , can be used throughout

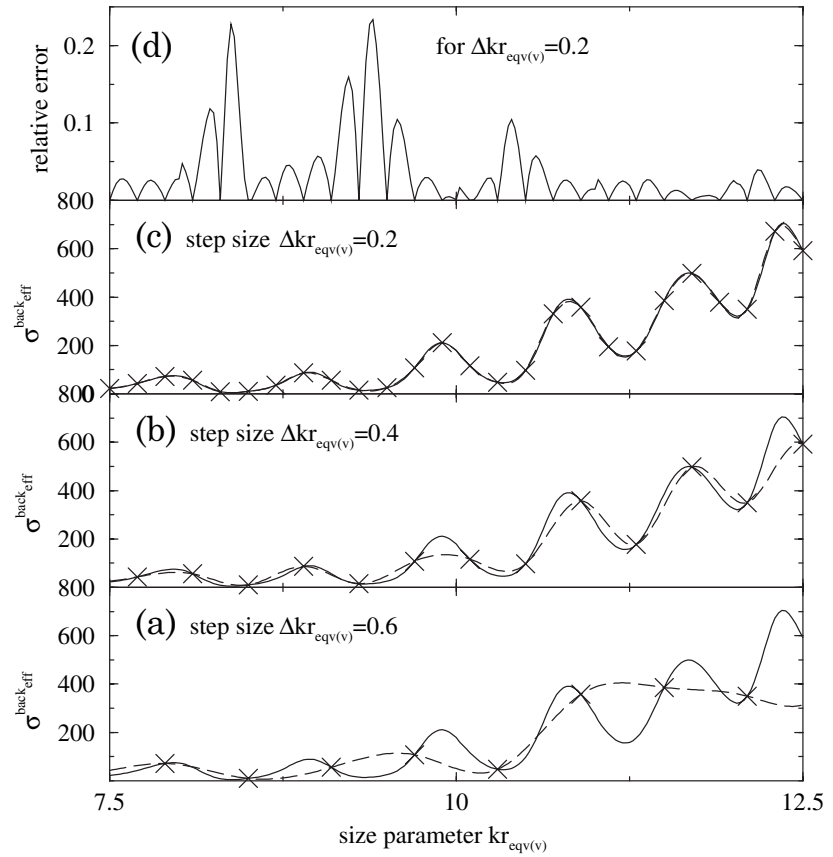

Fig. 2. Comparison of different resolutions of the backscattering efficiency $\sigma_{\text {dir.eff }}^{\text {back }}$ as a function of the volume-equivalent size parameter $k r_{\text {eqv }(v)}$ within the range [7.5,12.5] for a spherical scatterer with $n=1.33$. The solid curves in Figs. 2(a)-2(c) represent the reference with a size parameter resolution $\Delta \overline{k r_{\text {eqv }(v)}}=0.025$. The crosses are the points at which the computations have been performed for the different resolutions $\Delta k r_{\text {eqv }(v)}=(0.6,0.4,0.2)$, and the corresponding dashed curves are their cubic spline interpolations. The relative error of the interpolation of Fig. $\underline{2(\mathrm{c})}$ is shown in Fig. $2(\mathrm{~d})$. in the chosen region up to 40 . Note that the corresponding interpolation is performed in the user interface (Section 4).

\section{B. Angular Resolution of the Phase Functions}

Table 2 shows the angular resolution used to generate the phase function data for the database. This resolution was chosen again in such a way that a reliable interpolation of the phase functions with respect to the scattering angle is possible. At first, the whole size parameter range was divided into the two regions $(0,20]$ and $(20,40]$ since the phase function of any scatterer is in general smoother at lower size parameters than at higher ones. Then the angular resolution was investigated separately in each range. This allowed us to optimize the numerical effort because the computational time depends on the chosen angular resolution that is due to our convergence strategy. In both regions the resolution was determined at the highest size parameter as the worst case, i.e., at $k r_{\text {eqv }(v)}=20$ and $k r_{\text {eqv }(v)}=40$. Furthermore the phase functions of spheres oscillate much more than those of randomly oriented spheroids. Thus the investigations were done on spheres again. The resolution obtained in this case was also taken for the spheroids.

In view of different applications of the database which may require higher resolutions in certain angular regions we also divided the scattering plane into three regions, a forward scattering region $\left[0^{\circ}\right.$, $10^{\circ}$, a backscattering region $\left[173^{\circ}, 180^{\circ}\right]$, and a side scattering region $\left[10^{\circ}, 173^{\circ}\right]$. In this way forward or backscattering peaks can be better resolved, for example, which is important in transmission measurements or lidar applications. Examples of the investigations concerning the angular resolution and interpolation of the phase function are provided in Figs. 3 and 4 . The maximum errors were only reached in the deep troughs and amount to about $16 \%$ and $20 \%$, respectively. Note that the corresponding interpolation is again performed in the user interface.

\section{User Interface to the Database}

All data in the database are arranged with respect to their refractive indices and aspect ratios in a file system. Of course, they are accessible via the files in the directory tree. But this way is rather cumbersome, in general, due to the number of data sets, about 59,000. Therefore a user interface was developed to facilitate the data access. The user interface also provides some additional functionalities going beyond the simple readout of data. These are consistency tests

Table 2. Angular Resolution of the Phase Function Depending on the Volume-Equivalent Size Parameter $k r_{\text {eqv }(v)}$ and the Region of the Scattering Angle $\theta$

\begin{tabular}{lccc}
\hline & $\theta \in\left[0^{\circ}, 10^{\circ}\right]$ & $\theta \in\left[10^{\circ}, 173^{\circ}\right]$ & $\theta \in\left[173^{\circ}, 180^{\circ}\right]$ \\
\hline$k r_{\text {eqv }(v)} \in(0,20]$ & $0.75^{\circ}$ & $2.5^{\circ}$ & $0.75^{\circ}$ \\
$k r_{\text {eqv }(v)} \in(20,40]$ & $0.5^{\circ}$ & $1.5^{\circ}$ & $0.5^{\circ}$ \\
\hline
\end{tabular}




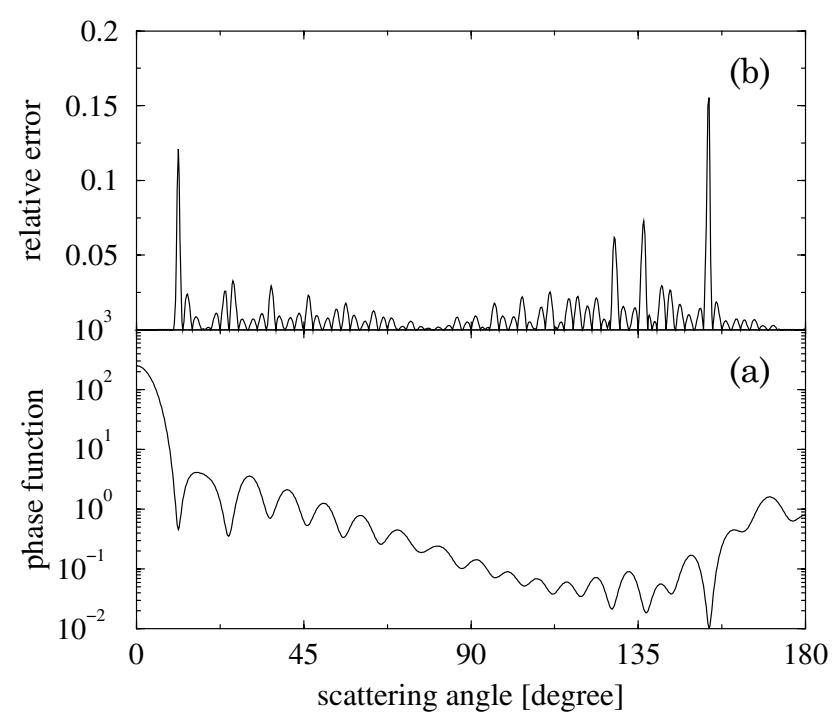

Fig. 3. (a) Phase function $p(\theta)$ of a sphere $\left(n=1.6, k r_{\text {eqv }(v)}=20\right)$ at an angular resolution of $0.25^{\circ}$ An approximation of this phase function is obtained if started from a lower resolution of $0.75^{\circ}$ in the forward and backward direction and $2.5^{\circ}$ in the side scattering direction and interpolated via cubic splines to the higher resolution of $0.25^{\circ}$. The resulting relative error is plotted in (b).

of the users requests, interpolations between data and the computation of size-averaged scattering quantities. The latter two functionalities are accomplished in different operation modes described below. They are intended for supporting a variety of applications of the database such as in radiative transfer simulations or in lidar inversion routines.

\section{A. First Operation Mode: Generating a Single Data Set}

In this mode the user can search for one specific data set of a randomly oriented spheroidal particle

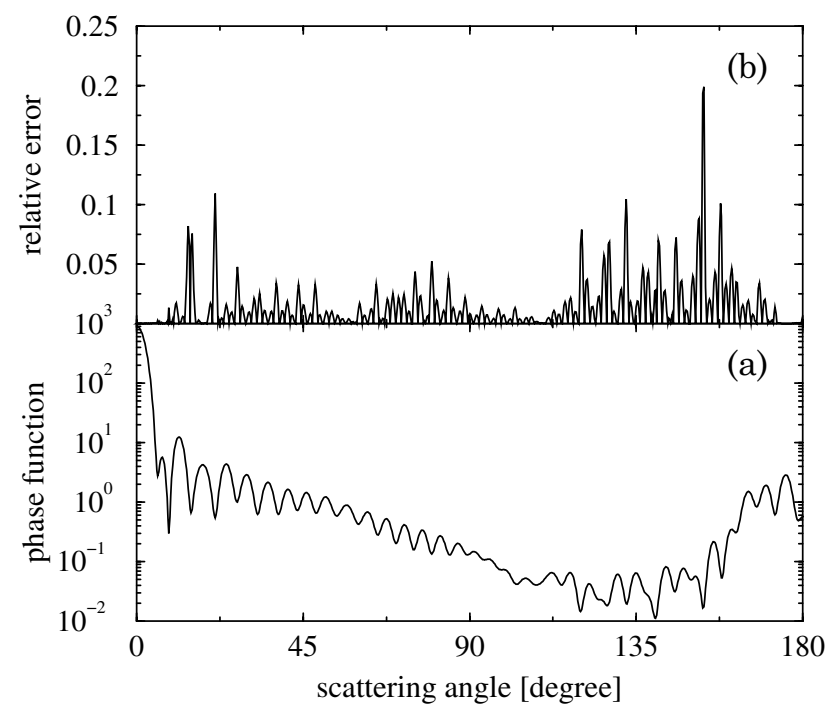

Fig. 4. (a) Phase function $p(\theta)$ of a sphere $\left(n=1.6, k r_{\text {eqv }(v)}=40\right)$ at an angular resolution of $0.25^{\circ}$. An approximation of this phase function is obtained if started from a lower resolution of $0.5^{\circ}$ in the forward and backward direction and $1.5^{\circ}$ in the side scattering direction and interpolated via cubic splines to the higher resolution of $0.25^{\circ}$. The resulting relative error is plotted in (b). characterized by the radius $r_{\mathrm{eqv}(v)}$ of the volumeequivalent sphere, the aspect ratio $a v$, the real part and the imaginary part of the complex refractive index $n$. Additionally to these parameters the frequency has to be fixed by the user so that the corresponding volume-equivalent size parameter $k r_{\text {eqv }(v)}$ can be computed internally. In general, this size parameter does not match any size parameter for which data are stored in the database. This is due to the finite resolution of all scattering quantities with respect to $k r_{\mathrm{eqv}(v)}$. But to allow for continuously varying scattering data with respect to $r_{\mathrm{eqv}(v)}$ or $k r_{\text {eqv }(v)}$, a linear interpolation scheme is used.

If $k r_{l}$ and $k r_{u}$ are the neighboring size parameters of the chosen $k r_{\operatorname{eqv}(v)}$ with $k r_{l} \leq k r_{\operatorname{eqv}(v)} \leq k r_{u}$ and if we indicate the corresponding scattering quantities with the indices $l$ ("lower") and $u$ ("upper"), respectively, then any total cross section $\sigma=\left(\sigma^{\text {ext }}, \sigma^{\text {scat }}\right.$, $\left.\sigma^{\text {abs }}, \sigma_{\text {dir }}^{\text {back }}, \sigma_{x}^{\text {back }}\right)$ belonging to the size parameter $k r_{\text {eqv }(v)}$ is linearly interpolated according to

$$
\sigma=w_{l} \cdot \sigma_{l}+w_{u} \cdot \sigma_{u} .
$$

Here the weighting functions $w_{l}$ and $w_{u}$ of the linear interpolation are given by

$$
\begin{gathered}
w_{l}=\frac{k r_{u}-k r_{\mathrm{eqv}(v)}}{k r_{u}-k r_{l}}, \\
w_{u}=\frac{k r_{\mathrm{eqv}(v)}-k r_{l}}{k r_{u}-k r_{l}} .
\end{gathered}
$$

They take the "distance" of the desired data set to the adjacent ones, which are present in the database, into account. Note that the corresponding efficiencies are simply obtained by dividing the cross sections [Eq. (20)] by the average geometrical cross section $q$. The single scattering albedo follows from the interpolated scattering and extinction cross sections. The phase function and the asymmetry parameter are interpolated according to

$$
\begin{array}{r}
p(\theta)=\frac{w_{l} \cdot \sigma_{l}^{\text {scat }} \cdot p_{l}(\theta)+w_{u} \cdot \sigma_{u}^{\text {scat }} \cdot p_{u}(\theta)}{\sigma^{\text {scat }}}, \\
g=\frac{w_{l} \cdot \sigma_{l}^{\text {scat }} \cdot g_{l}+w_{u} \cdot \sigma_{u}^{\text {scat }} \cdot g_{u}}{\sigma^{\text {scat }}} .
\end{array}
$$

In this mode the user has also the possibility to alter the given angular resolution of the phase function. If requested then a cubic spline interpolation is started, in general, to interpolate between the discrete data of the database.

The data set generated by the user interface in this mode contains the orientation-averaged scattering quantities, listed in Section 2, of the chosen spheroid. Additionally the total cross sections $\sigma^{\text {ext }}, \sigma^{\text {scat }}, \sigma^{\text {abs }}$, $\sigma_{\text {dir }}^{\text {back }}$, and $\sigma_{x}^{\text {back }}$ are provided. 


\section{B. Second Operation Mode: Generating a Range of Data}

In this mode scattering quantities for a certain parameter range are generated. The quantities are stored in the respective files as a function of the effective radius $r_{\mathrm{eqv}(v)}$, a function of the effective radius $r_{\mathrm{eqv}(v)}$, and the aspect ratio $a v$, or as a function of the effective radius $r_{\mathrm{eqv}(v)}$ and the real part of the refractive index $n$. Alternatively $r_{\text {eqv }(v)}$ can be replaced by the volume-equivalent size parameter $k r_{\mathrm{eqv}(v)}$. Moreover the user has to decide which orientation-averaged scattering quantity is provided. Possible choices are

- one of the efficiencies
$\left.\sigma_{\text {dirk }}^{\text {back }}, \sigma_{x \text { eff }}^{\text {back }}\right)$,

- one of the cross sections $\left(\sigma^{\text {ext }}, \sigma^{\text {scat }}, \sigma^{\text {abs }}, \sigma_{\text {dir }}^{\text {back }}\right.$, $\left.\sigma_{x}^{\text {back }}\right)$

- the single scattering albedo $\omega$,

- the asymmetry parameter $g$,

- the lidar ratio $S=\sigma^{\text {ext }} /\left(\sigma_{\text {dir }}^{\text {back }}+\sigma_{x}^{\text {back }}\right)$,

- the direct-polarized backscattering coefficient $\sigma^{\text {dir,back }}=\sigma_{\text {dir }}^{\text {back }} /\left(\sigma_{\text {dir }}^{\text {back }}+\sigma_{x}^{\text {back }}\right)$, or

- the cross-polarized backscattering coefficient $\sigma^{x, \text { back }}=\sigma_{x}^{\text {back }} /\left(\sigma_{\text {dir }}^{\text {back }}+\sigma_{x}^{\text {back }}\right)$.

Note that they are taken directly from the database and computed internally in the user interface, respectively, without any size parameter interpolation.

\section{Third Operation Mode: Generating an Averaged Data} Set

In this mode the user interface allows to calculate the size and aspect ratio average of all scattering quantities of the first mode (Subsection 4.A). In doing so the factorized distribution function

$$
N\left(r_{\mathrm{eqv}(v)}, a v\right)=N^{r}\left(r_{\mathrm{eqv}(v)}\right) \cdot N^{a v}(a v)
$$

is used. Here $N^{r}\left(r_{\mathrm{eqv}(v)}\right)$ is one of the following size distribution functions predefined in the user interface:

- modified gamma distribution

$$
N^{r}\left(r_{\mathrm{eqv}(v)}\right)=r_{\mathrm{eqv}(v)}^{\alpha} \cdot \exp \left(-\frac{\alpha \cdot r_{\mathrm{eqv}(v)}^{\gamma}}{\gamma \cdot r_{c}^{\gamma}}\right)
$$

- monomodal log-normal distribution

$$
N^{r}\left(r_{\mathrm{eqv}(v)}\right)=r_{\mathrm{eqv}(v)}^{-1} \cdot \exp \left[-\frac{\left(\ln r_{\mathrm{eqv}(v)}-\ln r_{1}\right)^{2}}{2 \ln ^{2} \sigma_{1}}\right],
$$

- or modified power law distribution

$$
N^{r}\left(r_{\text {eqv }(v)}\right)=\left\{\begin{array}{cc}
1, & 0 \leq r_{\text {eqv }(v)} \leq r_{1} \\
\left(r / r_{1}\right)^{\alpha}, & r_{1} \leq r_{\text {eqv }(v)} \leq r_{2} \\
0, & r_{2}<r_{\text {eqv }(v)}
\end{array}\right.
$$

The parameters of the different size distribution functions have to be specified by the user on request. A discrete aspect ratio distribution functions $N^{a v}(a v)$ of the form

$$
N^{a v}(a v)=\left\{\begin{array}{c}
N_{1}^{a v}=N^{a v}(0.67) \\
N_{2}^{a v}=N^{a v}(0.77) \\
\vdots \\
N_{7}^{a v}=N^{a v}(1.5)
\end{array}\right.
$$

is assumed. $N_{i}^{a v}$ represents the number of particles with the corresponding aspect ratio given in Table 1 . The user has to provide these numbers on request. Any averaged scattering quantity $X$ is then calculated according to

$$
\begin{aligned}
\langle X\rangle_{r, a v}= & \frac{1}{\langle N\rangle_{r, a v}} \sum_{i} N_{i}^{a v} \cdot \int N^{r}\left(r_{\operatorname{eqv}(v)}\right) \\
& \cdot X\left(r_{\operatorname{eqv}(v)}, a v\right) \mathrm{d} r_{\operatorname{eqv}(v)}
\end{aligned}
$$

with

$$
\langle N\rangle_{r, a v}=\sum_{i} N_{i}^{a v} \cdot \int N^{r}\left(r_{\operatorname{eqv}(v)}\right) \mathrm{d} r_{\operatorname{eqv}(v)} .
$$

The above integration is performed in applying an Eulerian integration rule with a step size defined by the user.

\section{Examples}

In the following we want to present some examples to illustrate the usage of the database in the different operation modes and its capabilities. Figure 5 shows a typical application of the first operation mode in Subsection 4.A. We are looking for the phase function of a randomly oriented spheroid with $n=1.8, a v=$ 1.5 , and $k r_{\text {eqv }(v)}=39.52$. Since this is not present in the database it is linearly interpolated by use of Eq. (23) between the phase functions belonging to the size parameters $k r_{\text {eqv }(v)}=39.4$ and $k r_{\text {eqv }(v)}=$ 39.6, which exist in the database. The relative error of the interpolated phase function to the one calculated at the desired size parameter [in the present example $k r_{\text {eqv }(v)}=39.52$ ] is shown in Fig. 5(b). It is seen that the relative error does not exceed $3 \%$ in the forward scattering direction up to a scattering angle of about $50^{\circ}$. The maximum error amounts to about $15 \%$ and is obtained only in deepest trough of the phase function. Thus this example demonstrates two important things. First, let us recall that the size parameter resolution $\Delta k r_{\text {eqv }(v)}=0.2$ in the underlying database was originally determined by investigating the backscattering efficiencies of spheres (see Subsection 3.A). Using this resolution it is seen that it is also possible to linearly interpolate phase functions of randomly oriented spheroids within a sufficient accuracy for most applications. Second, the resolution was fixed within the size parameter 


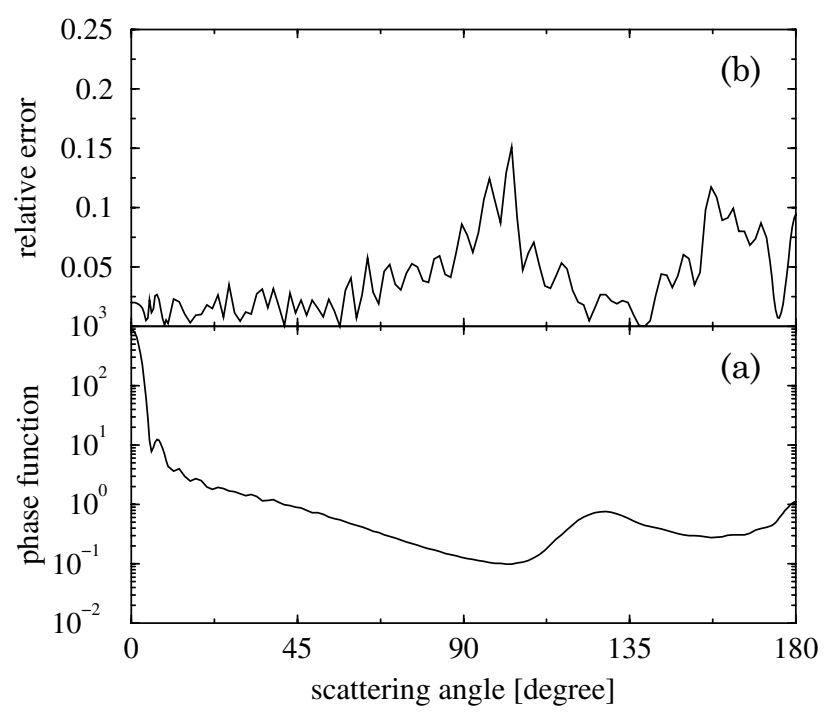

Fig. 5. (a) Phase functions $p(\theta)$ of a randomly oriented spheroid with $n=1.8, a v=1.5$, and $k r_{\text {eqv }(v)}=39.52\left(r_{\text {eqv }(v)}=2.23 \mu \mathrm{m}\right)$ linearly interpolated by use of Eq. (23) between the volume-equivalent size parameters $k r_{\text {eqv }(v)}=39.4$ and $k r_{\text {eqv }(v)}=39.6$. The relative error of this phase function to the one calculated at the desired size parameter $k r_{\text {eqv }(v)}=39.52$ is shown in (b).

range up to 12.5 (see again Subsection 3.A). It turns out that it can be used up to $k r_{\text {eqv }(v)}=40$.

Figure $\underline{6}$ shows three examples belonging to the second operation mode in Subsection 4.B. These are the backscattering efficiency $\sigma_{\text {dir.eff }}^{\text {back }}+\sigma_{x, \text { eff }}^{\text {back }}$, the extinction efficiency $\sigma_{\text {eff }}^{\text {ext }}$, and the lidar ratio $S$ of a randomly oriented oblate spheroid with $a v=0.67$ (dotted curves), a sphere (solid curves), and of a prolate spheroid with $a v=1.5$ (dashed curves), all

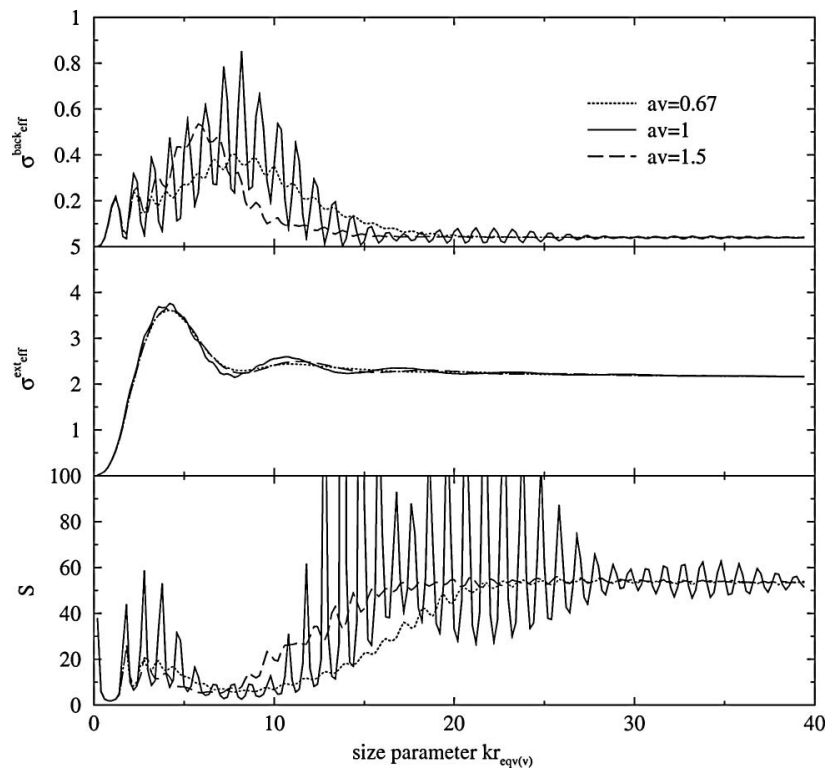

Fig. 6. Backscattering efficiency $\sigma_{\text {dir.eff }}^{\text {back }}+\sigma_{x \text {,eff }}^{\text {back }}$, extinction efficiency $\sigma_{\text {eff }}^{\text {ext }}$, and lidar ratio $S$ of a randomly oriented oblate spheroid with $a v=0.67$ (dotted curves), a sphere (solid curves), and a prolate spheroid with $a v=1.5$ (dashed curves), all having a refractive index $n=1.5+i \cdot 0.05$, as a function of the volume-equivalent size parameter $k r_{\operatorname{eqv}(v)}$.

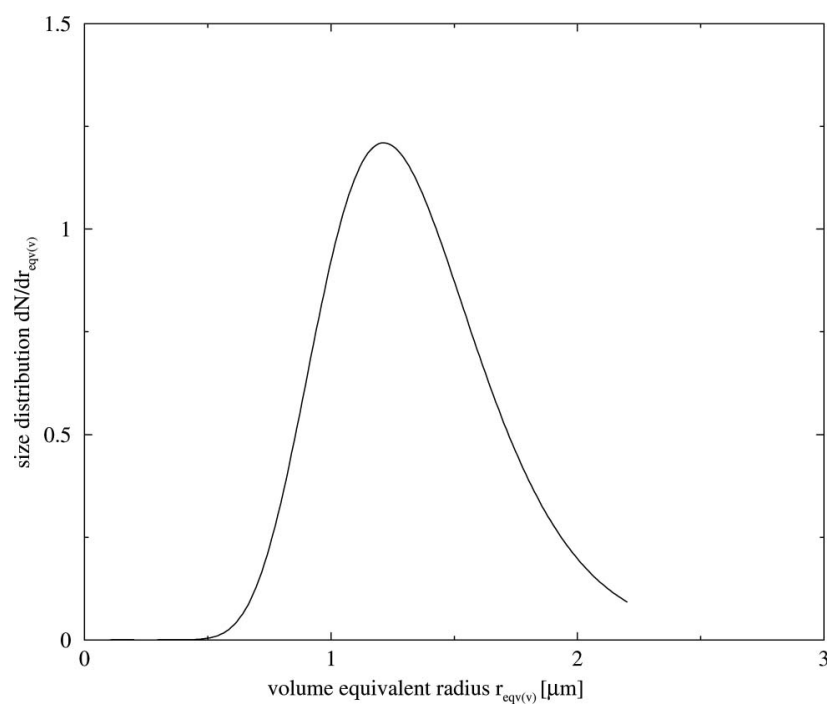

Fig. 7. Monomodal log-normal size distribution function (27) with $r_{1}=1.3 \mu \mathrm{m}$ and $\sigma_{1}=1.3$.

having a refractive index $n=1.5+i \cdot 0.05$, as a function of the volume-equivalent size parameter $k r_{\text {eqv }(v)}$. These quantities play an important role in lidar experiments. One aim of such experiments is to characterize cloud and aerosol particles in terms of their shape by distinguishing between spherical and nonspherical ones (e.g., Sassen [17]). It is seen that the extinction efficiency is not sensitive with respect to the particle shapes chosen. In contrast to this the backscattering efficiency and the lidar ratio reveal a quite different behavior for the different particle shapes.

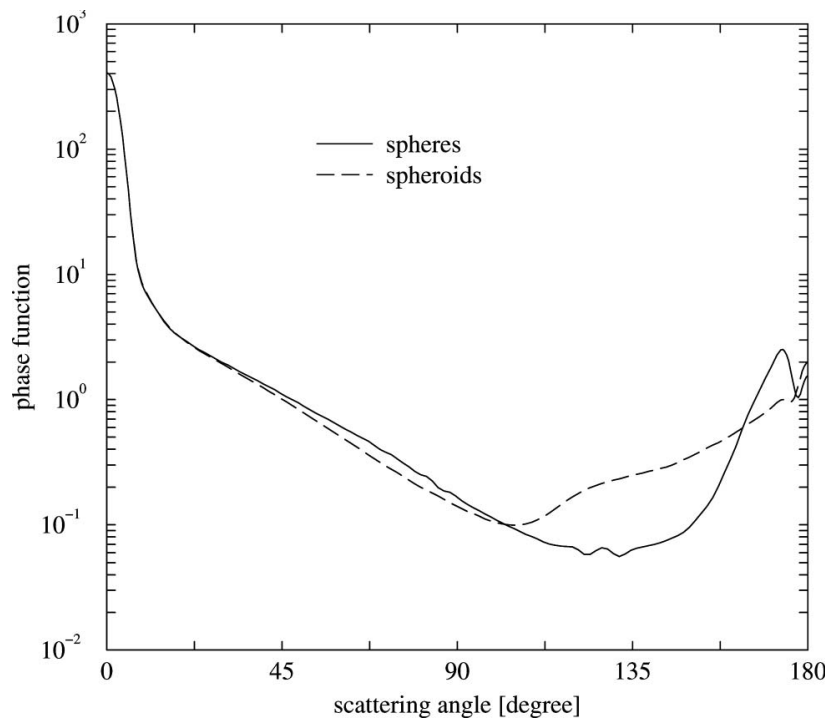

Fig. 8. Phase functions $p(\theta)$ of spheres (solid line) and randomly oriented spheroids (dashed line) averaged over the particle size in using the size distribution function of Fig. $\underline{7}$ at a wavelength $\lambda=$ $0.355 \mu \mathrm{m}$ (volume-equivalent size parameter up to 40). All aspect ratios of Table 1 with the same weight are present in the mixture of the spheroids. The scatterers have a refractive index $n=1.6$ throughout. 


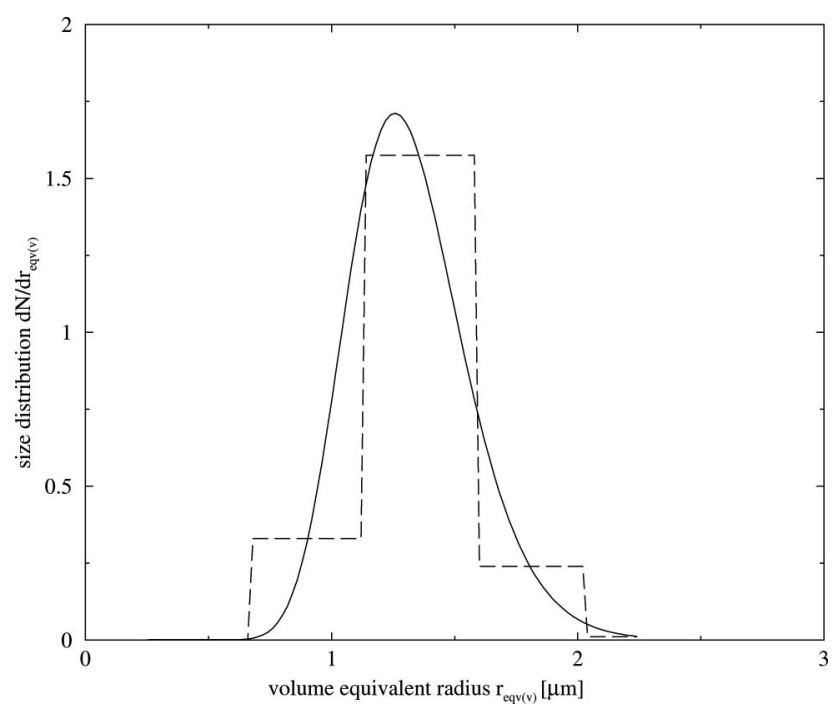

Fig. 9. Monomodal log-normal size distribution function (27) with $r_{1}=1.3 \mu \mathrm{m}$ and $\sigma_{1}=1.2$ (solid line) and a rough discretization of this distribution function to a size class distribution function (dashed line).

In order to present an example of the third operation mode in Subsection 4.C the monomodal lognormal size distribution function of Fig. 7 was used to compute phase functions of mixtures of spheres and randomly oriented spheroids. In doing so all aspect ratios of Table 1 with the same weight were taken into account in the mixture of the spheroids. Furthermore all scatterers have a refractive index $n=1.6$ at a wavelength $\lambda=0.355 \mu \mathrm{m}$. Figure $\underline{8}$ shows both normalized phase functions $p(\theta)$. One basic effect of nonspherical particles-the enhanced side

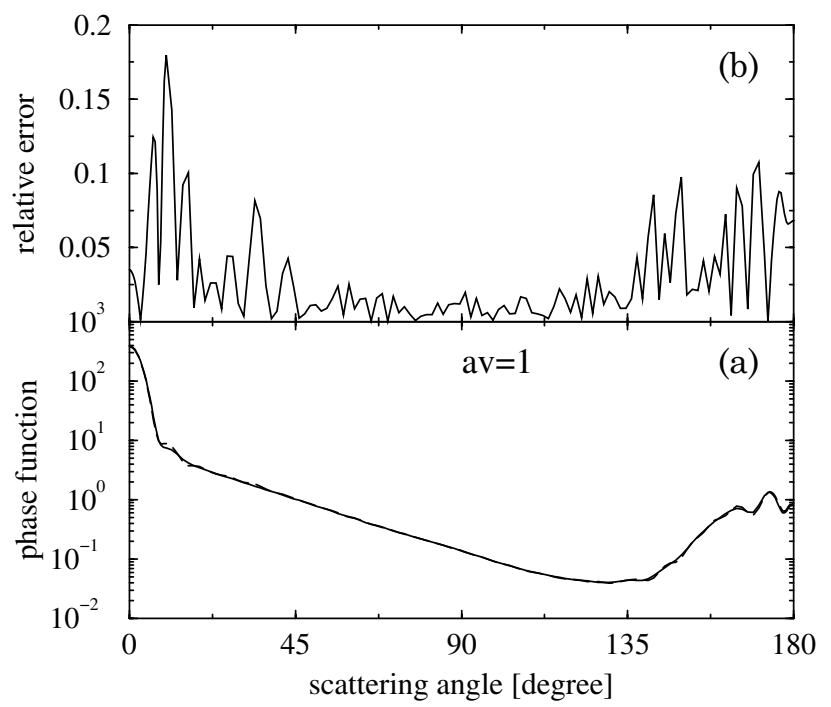

Fig. 10. Phase functions $p(\theta)$ of spheres averaged over the particle size in using the monomodal log-normal size distribution function of Fig. 9 [solid line in (a)] and the size class distribution function of Fig. 9 [dashed line in (a)]. The spheres have a refractive index $n=1.5+i \cdot 0.001$ at a wavelength $\lambda=0.355 \mu \mathrm{m}$ (volumeequivalent size parameter up to 40 ). In (b) the relative error of the phase function based on the size class distribution function is shown.

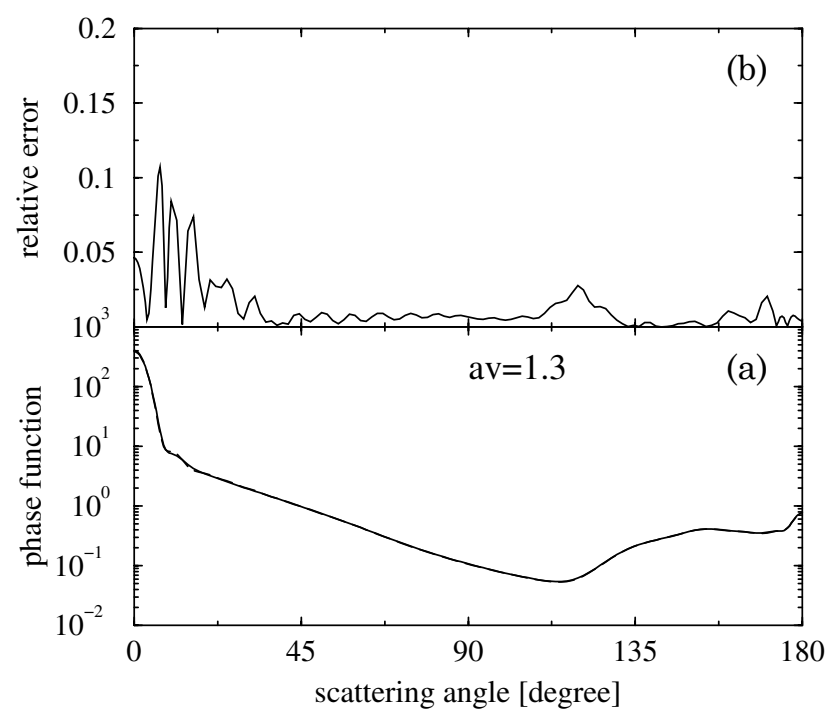

Fig. 11. Phase functions $p(\theta)$ of randomly oriented spheroids averaged over the particle size in using the monomodal log-normal size distribution function of Fig. 9 [solid line in (a)] and the size class distribution function of Fig. $\overline{9}$ [dashed line in (a)]. The spheroids have an aspect ratio $a v=1.3$ and a refractive index $n=1.5+$ $i \cdot 0.001$ at a wavelength $\lambda=0.355 \mu \mathrm{m}$ (volume-equivalent size parameter up to 40). In (b) the relative error of the phase function based on the size class distribution function is shown.

scattering at about $120^{\circ}$ as compared to spherescan be clearly seen.

Another monomodal log-normal size distribution function (27) with $r_{1}=1.3 \mu \mathrm{m}$ and $\sigma_{1}=1.2$ and a rough discretization of this distribution function to a size class distribution function are plotted in Fig. $\underline{9}$.

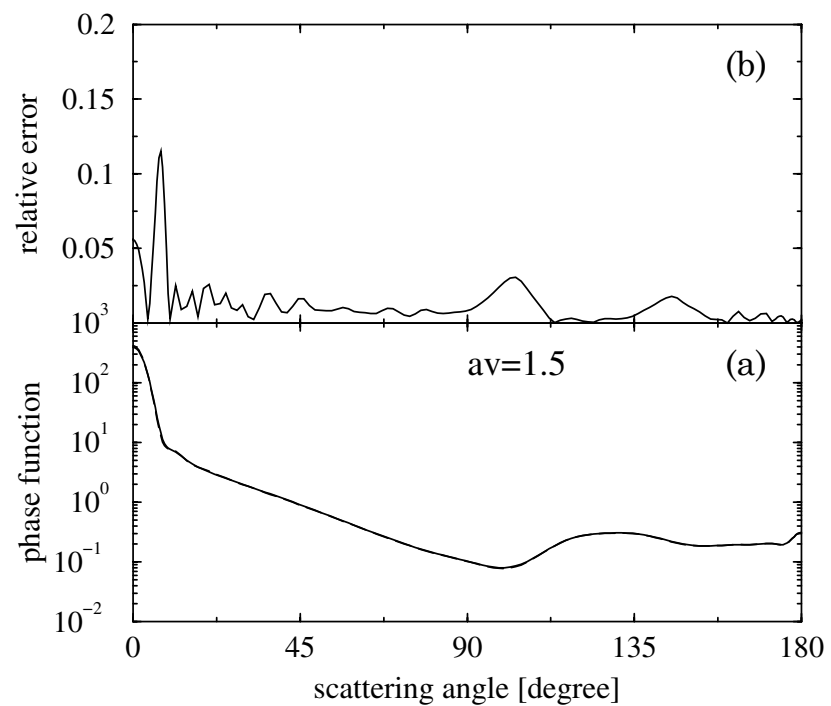

Fig. 12. Phase functions $p(\theta)$ of randomly oriented spheroids averaged over the particle size in using the monomodal log-normal size distribution function of Fig. $\underline{9}$ [solid line in (a)] and the size class distribution function of Fig. $\underline{\overline{9}}$ [dashed line in (a)]. The spheroids have an aspect ratio $a v=1.5$ and a refractive index $n=1.5+$ $i \cdot 0.001$ at a wavelength $\lambda=0.355 \mu \mathrm{m}$ (volume-equivalent size parameter up to 40). In Fig. 12(b) the relative error of the phase function based on the size class distribution function is shown. 


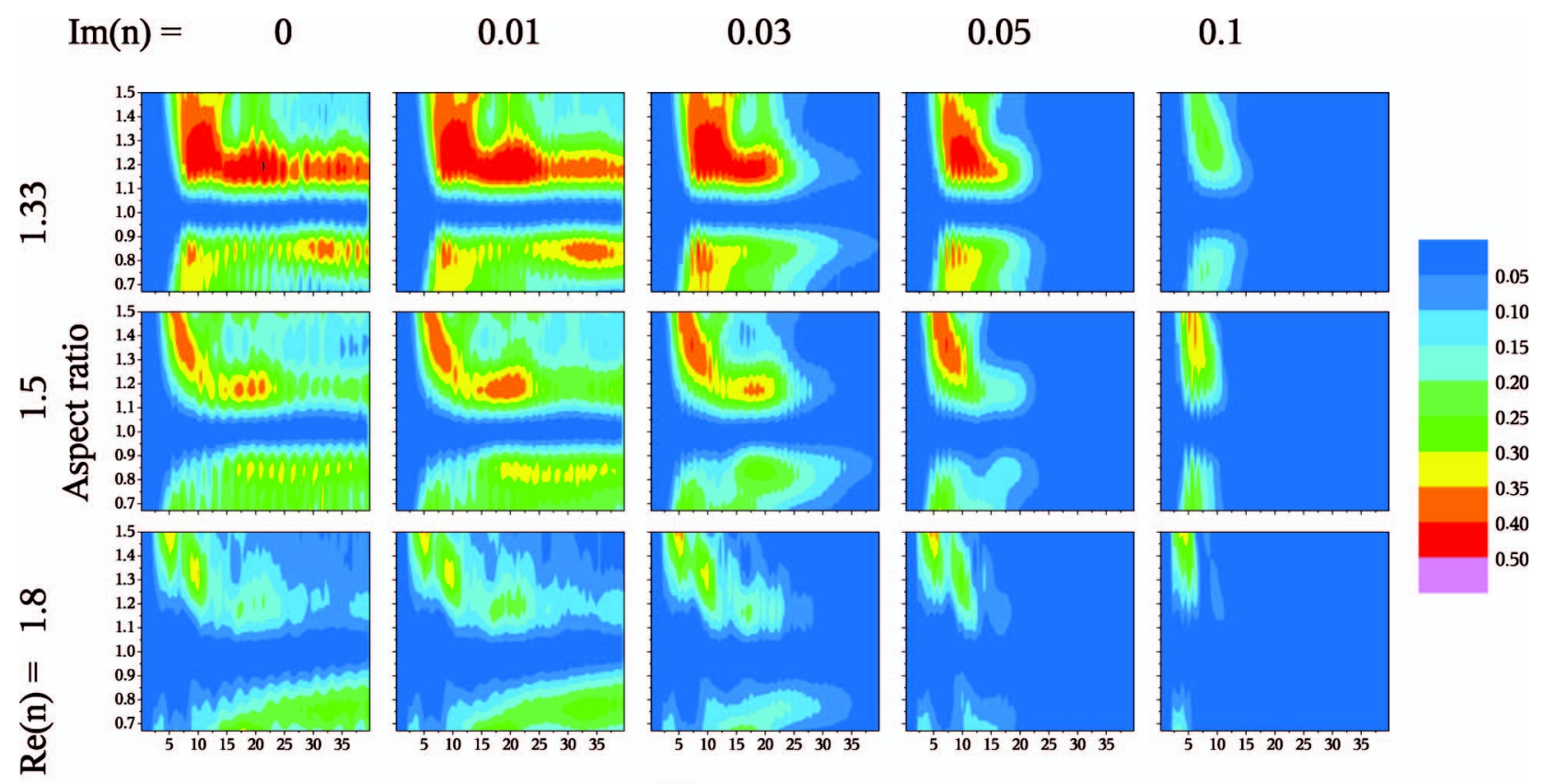

Size parameter

Fig. 13. Backscattering depolarization $\sigma_{x, \text { eff }}^{\text {back }} /\left(\sigma_{\text {dir,eff }}^{\text {back }}+\sigma_{x, \text { eff }}^{\text {back }}\right)$ as a function of the aspect ratio $a v$ and the volume-equivalent size parameter $k r_{\text {eqv }(v)}$ for different refractive indices $n$.

Both functions were used to investigate the influence of uncertainties in the knowledge of the size distribution function for computing size-averaged phase functions. Figures 10-12, provide examples of these investigations. The integrations over the size distributions were performed in applying an Eulerian rule with a step size of $0.02 \mu \mathrm{m}$. It is seen that the maximum error, when approximating the corresponding distribution function (27) by a size class distribution function, is about $18 \%$ in the case of sphere mixtures. The maximum error does not exceed $12 \%$ for prolate spheroid mixtures. Such an accuracy is sufficient for many applications. Thus the results obtained support the customary practice to use experimentally determined size class distribution functions for computing the scattering behavior of particles. On the other hand, this means that the size distribution function can be deduced from phase function
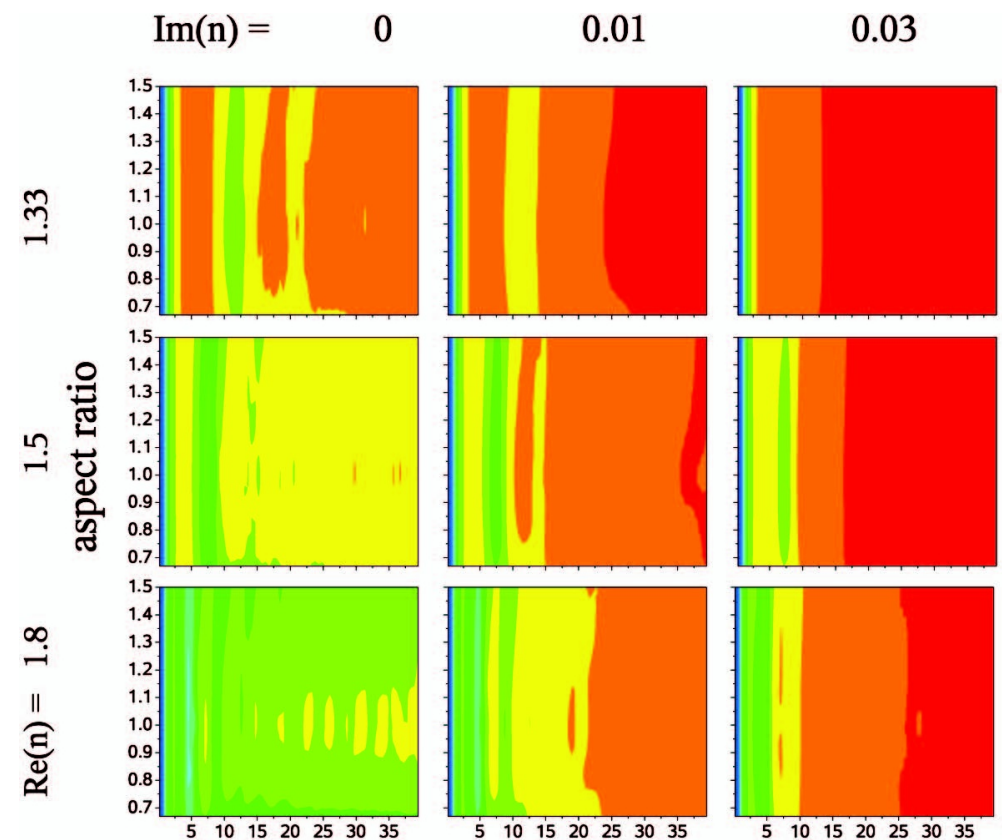

0.05

0.1
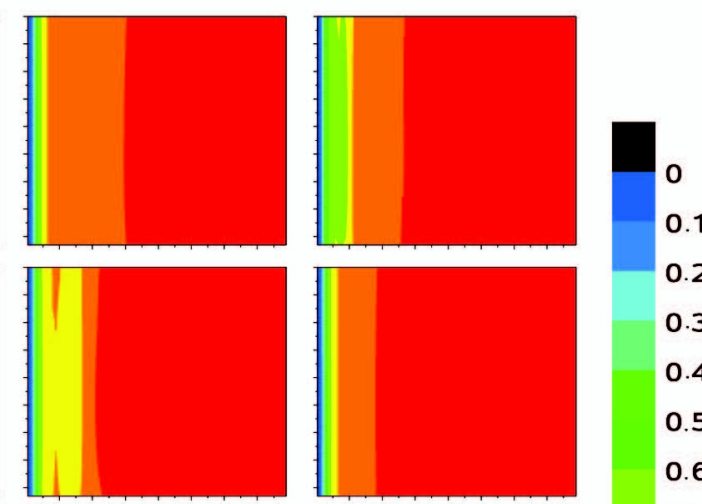

0.2

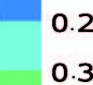

0.3

0.4

0.5

0.6
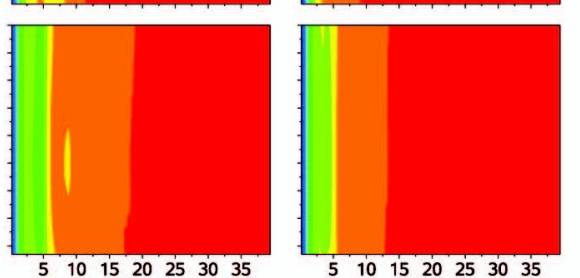

size parameter

Fig. 14. Asymmetry parameter $g$ as a function of the aspect ratio $a v$ and the volume-equivalent size parameter $k r_{\text {eqv }(v)}$ for different refractive indices $n$. 
measurements only up to a certain degree when all other particle parameters are known.

Figures 13 and 14 provide examples of scattering quantities over wide parameter ranges. Figure 13 shows the backscattering depolarization $\sigma_{x, \text { eff }}^{\text {back }}$ $\left(\sigma_{\text {dir,eff }}^{\text {back }}+\sigma_{x, \text { eff }}^{\text {back }}\right)$ as a function of the aspect ratio $a v$ and the volume-equivalent size parameter $k r_{\text {eqv }}(v)$ for different refractive indices $n$. The bluish regions mark parameter sets with a lower depolarization, while the reddish ones designate larger depolarization values. In general, randomly oriented prolate spheroids $(a v>1)$ exhibit larger depolarizations than oblate ones $(a v<1)$. In each tile, both regions are clearly separated by the zero-depolarization band originating from the spheres $(a v=1)$. It is furthermore seen that, within the chosen parameter range, the largest depolarization differences occur for small real parts $(\operatorname{Re}(n)=1.33)$ and small imaginary parts $(\operatorname{Im}(n)=0-0.05)$ of the refractive index. Otherwise spherical and spheroidal particles become increasingly indistinguishable by means of backscattering depolarization measurements. The same holds also if the particles are small enough $\left(k r_{\text {eqv }(v)} \lesssim 2\right)$.

Figure 14 shows the asymmetry parameter $g$ as a function of the aspect ratio $a v$ and the size parameter $k r_{\text {eqv }(v)}$ for different refractive indices $n$. Large values of $g$, i.e., large differences between the forward and the backscattering region of the phase function, can be observed for stronger absorbing particles and larger size parameters, in general. This is due to the fact that the forward scattering peak increases with increasing size parameter while side and backscattering features are damped by absorption. Smaller scatterers with $k r_{\text {eqv }(v)} \lesssim 2$ yield the smallest asymmetry parameters.

Figures such as these give experimenters and modelers a quick overview to identify interesting regions for going deeper in their investigations, for designing new experiments or for planning future satellite missions. The expense to generate such plots is relatively low when using the database.

\section{Conclusions}

We have presented a database of light scattering quantities of randomly oriented dielectric spheroidal particles in the resonance region. A well-tested $T$ matrix method was used to generate the data. The data possess a defined accuracy so that they can be ideally used as benchmarks for electromagnetic and light scattering computations of spheroids. The chosen fine resolution with respect to the size parameter and the scattering angle enables reliable interpolations between the data to generate continuously varying scattering quantities with a sufficient accuracy. A user interface was developed to facilitate the data access and to process the data. On request, the database, including the documentation, is available, free of charge, on a CD-ROM.

\section{References}

1. K. M. Markowicz, P. J. Flatau, A. M. Vogelmann, P. K. Quinn, and E. J. Welton, "Clear-sky infrared aerosol radiative forcing at the surface and the top of the atmosphere," Q. J. R. Meteorol. Soc. 129, 2927-2947 (2003).

2. S. Otto, E. Bierwirth, B. Weinzierl, K. Kandler, M. Esselborn, M. Tesche, A. Schladitz, M. Wendisch, and T. Trautmann, "Solar radiative effects of a Saharan dust plume observed during SAMUM assuming spheroidal particles," Tellus B 61, 270-296 (2009).

3. Y. J. Kasai, J. Urban, C. Takahashi, S. Hoshino, K. Takahashi, J. Inatani, M. Shiotani, and H. Masuko, "Stratospheric ozone isotope enrichment studied by submillimeter wave heterodyne radiometry: the observation capabilities of SMILES," IEEE Trans. Geosci. Remote Sensing 44, 676693 (2006)

4. M. G. Mlynczak, D. G. Johnson, H. Latvakoski, K. Jucks, M. Watson, D. P. Kratz, G. Bingham, W. A. Traub, S. J. Wellard, C. R. Hyde, and X. Liu, "First light from the Far-Infrared Spectroscopy of the Troposphere (FIRST) instrument," Geophys. Res. Lett. 33, L07704 (2006).

5. C. Emde, S. A. Buehler, P. Eriksson, and T. R. Sreerekha, "The effect of cirrus clouds on microwave limb radiances," Atmos. Res. 72, 383-401 (2004).

6. P. Yang, H. Wei, H.-L. Huang, B. A. Baum, Y. X. Hu, G. W. Kattawar, M. I. Mishchenko, and Q. Fu, "Scattering and absorption property database for nonspherical ice particles in the near- through far-infrared spectral region," Appl. Opt. 44, 5512-5523 (2005).

7. O. Dubovik, A. Sinyuk, T. Lapyonok, B. N. Holben, M. Mishchenko, P. Yang, T. F. Eck, H. Volten, O. Munoz, B. Veihelmann, W. J. van der Zande, J.-F. Leon, M. Sorokin, and I. Slutsker, "Application of spheroid models to account for aerosol particle nonsphericity in remote sensing of desert dust," J. Geophys. Res. 111, D11208 (2006).

8. H. Volten, O. Munoz, J. W. Hovenier, J. F. de Haan, W. Vassen, W. J. van der Zande, and L. B. F. M. Waters, "WWW scattering matrix database for small mineral particles at 441.6 and 632.8 nm," J. Quant. Spectrosc. Radiat. Transfer 90, 191-206 (2005).

9. J. Wauer, K. Schmidt, T. Rother, T. Ernst, and M. Hess, "Two software tools for plane-wave scattering on nonspherical particles in the German Aerospace Center's virtual laboratory," Appl. Opt. 43, 6371-6379 (2004).

10. P. C. Waterman, "Symmetry, unitarity, and geometry in electromagnetic scattering," Phys. Rev. D 3, 825-839 (1971).

11. M. I. Mishchenko, "Light scattering by randomly oriented axially symmetric particles,” J. Opt. Soc. Am. A 8, 871-882 (1991).

12. M. I. Mishchenko, L. D. Travis, and A. A. Lacis, Scattering, Absorption, and Emission of Light by Small Particles (Cambridge University Press, 2002).

13. H. C. van de Hulst, Light Scattering by Small Particles (Wiley, 1957).

14. L. Tsang, J. A. Kong, and R. T. Shin, Theory of Microwave Remote Sensing (Wiley, 1985).

15. N. G. Khlebtsov, "Orientational averaging of light-scattering observables in the T-matrix approach," Appl. Opt. 31, 5359-5365 (1992).

16. P. W. Barber and S. C. Hill, Light Scattering by Particles: Computational Methods (World Scientific, 1990).

17. K. Sassen, "lidar backscatter depolarization technique for cloud and aerosol research," in Light Scattering by Nonspherical Particles, M. I. Mishchenko, J. W. Hovenier, and L. D. Travis, eds. (Academic, 2000), pp. 393-416. 